\title{
New Contribution to the Advancement of Fixed Point Theory, Equilibrium Problems, and Optimization Problems 2014
}

\author{
Wei-Shih Du, ${ }^{1}$ Erdal Karapinar, ${ }^{2,3}$ Lai-Jiu Lin, ${ }^{4}$ and Gue Myung Lee ${ }^{5}$ \\ ${ }^{1}$ Department of Mathematics, National Kaohsiung Normal University, Kaohsiung 824, Taiwan \\ ${ }^{2}$ Department of Mathematics, Atilim University, Incek, 06836 Ankara, Turkey \\ ${ }^{3}$ Nonlinear Analysis and Applied Mathematics Research Group (NAAM), King Abdulaziz University, Jeddah, Saudi Arabia \\ ${ }^{4}$ Department of Mathematics, National Changhua University of Education, Changhua 50058, Taiwan \\ ${ }^{5}$ Department of Applied Mathematics, Pukyong National University, Busan 608-737, Republic of Korea
}

Correspondence should be addressed to Wei-Shih Du; wsdu@nknucc.nknu.edu.tw

Received 20 November 2014; Accepted 20 November 2014

Copyright (c) 2015 Wei-Shih Du et al. This is an open access article distributed under the Creative Commons Attribution License, which permits unrestricted use, distribution, and reproduction in any medium, provided the original work is properly cited.

The special issue entitled "New Contribution to the Advancement of Fixed Point Theory, Equilibrium Problems, and Optimization Problems" was published by Journal of Applied Mathematics in 2013. After this special issue, the editorial team received several letters from a number of authors and publishers in which they asked to repeat it. Under the continuous demands of authors and reviewers, the editorial team considered opening this special issue with the same title and content.

As in the first issue, we focused on fixed point theory due to its wide application potential in various branches of pure and applied sciences, such as physics, engineering, chemistry, biology, economics, and computer science. We aimed to publish recent developments in both fixed point theory and optimization theory together with the applications on possible real world problems.

We received 44 papers in the interdisciplinary research fields of nonlinear sciences. This special issue includes only 6 high-quality peer-reviewed articles that contribute new innovative techniques and novel ideas to deal with different aspects of fixed point theory, equilibrium problems, and optimization problems. Evidently, it is not possible to adequately represent in this special issue all directions of current research in fixed point theory, equilibrium problems, and optimization problems. However, we believe that these articles will motivate and inspire further scientific activities in the field of fixed point theory, optimization, and their applications.

\section{Acknowledgments}

We would like to express our deepest gratitude to many authors and reviewers who contributed so greatly to the success of this special issue. In addition, we would also like to convey our appreciation to the editorial board members of this journal, for their kind assistance and support throughout the reviewing process and the preparation of this special issue.

Wei-Shih Du

Erdal Karapinar

Lai-Jiu Lin

Gue Myung Lee 


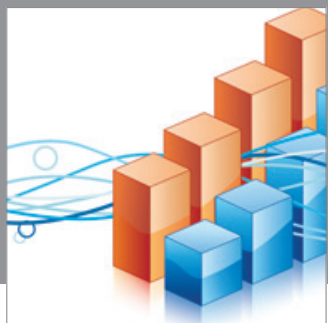

Advances in

Operations Research

mansans

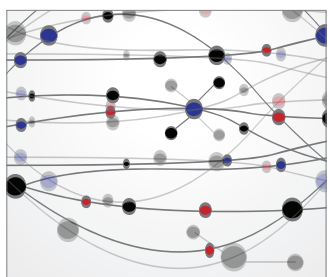

The Scientific World Journal
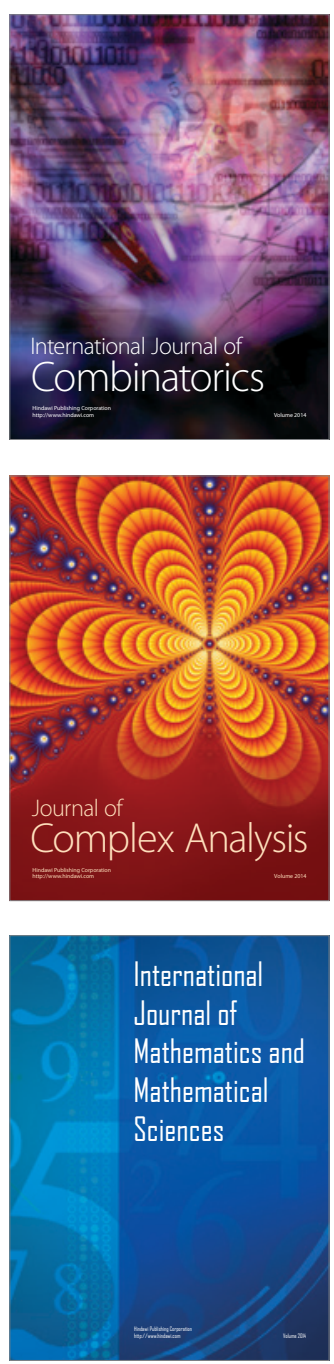
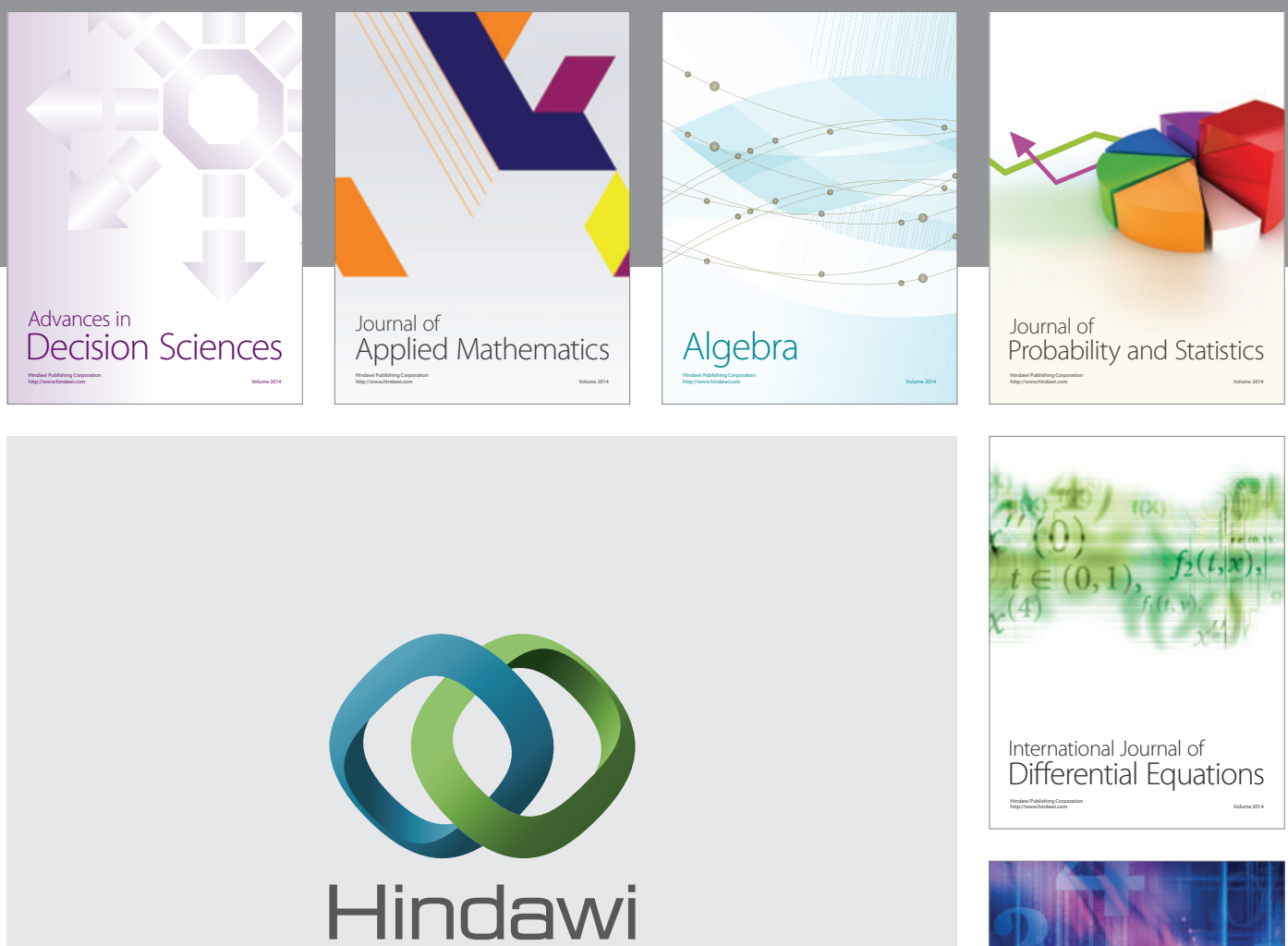

Submit your manuscripts at http://www.hindawi.com
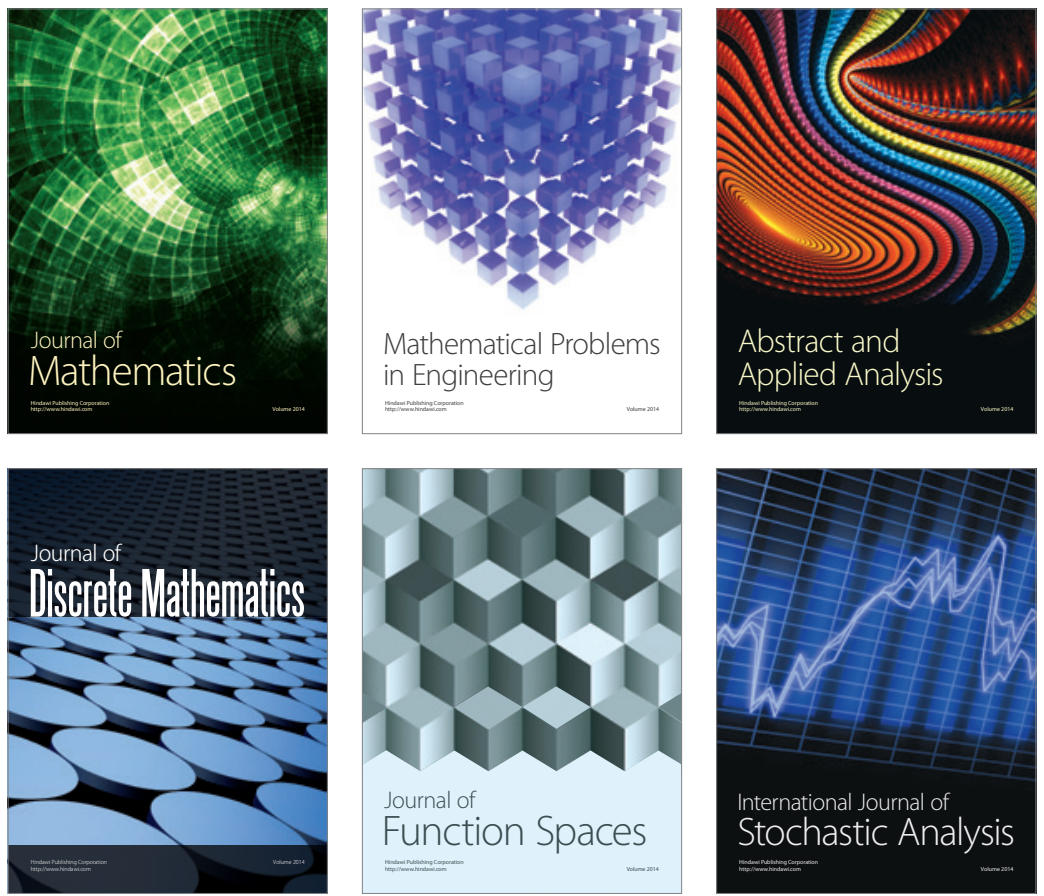

Journal of

Function Spaces

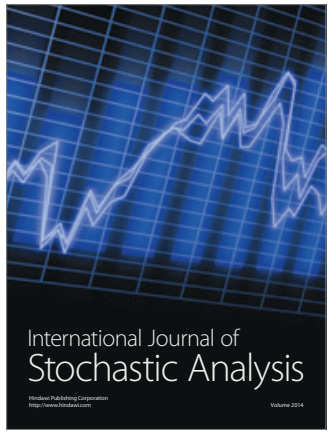

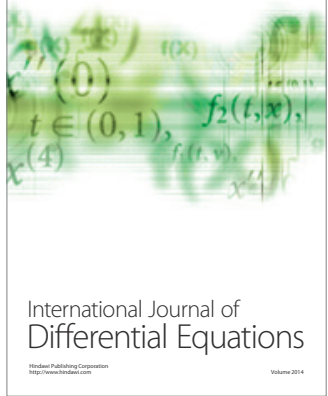
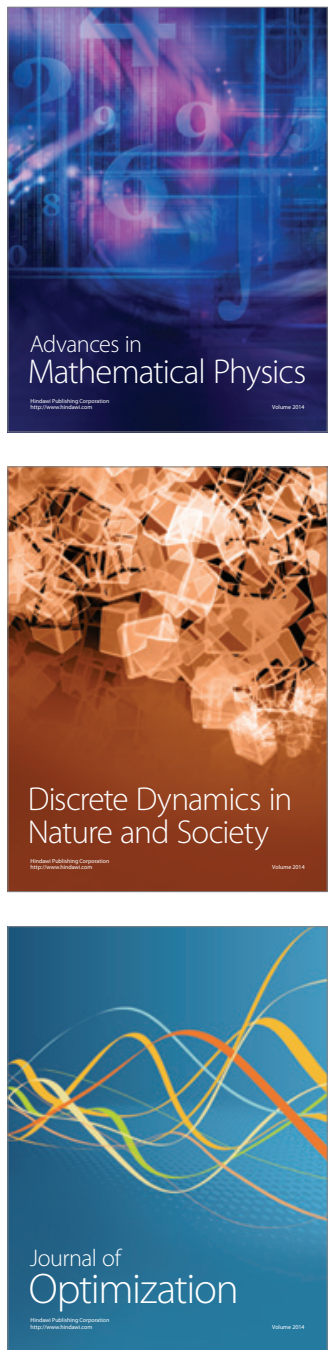